\title{
Article
}

\section{Some results on dependent random variables and a connection with the multivariate s-increasing convex order}

\author{
Hadjikyriakou, Milto \\ Available at http://clok.uclan.ac.uk/17761/ \\ Hadjikyriakou, Milto ORCID: 0000-0001-5672-7792 (2017) Some results on \\ dependent random variables and a connection with the multivariate $s$ - \\ increasing convex order. Communication in Statistics -Theory and Methods . \\ ISSN 0361-0926
}

It is advisable to refer to the publisher's version if you intend to cite from the work. http://dx.doi.org/10.1080/03610926.2017.1291977

For more information about UCLan's research in this area go to http://www.uclan.ac.uk/researchgroups/ and search for <name of research Group>.

For information about Research generally at UCLan please go to http://www.uclan.ac.uk/research/

All outputs in CLoK are protected by Intellectual Property Rights law, including Copyright law. Copyright, IPR and Moral Rights for the works on this site are retained by the individual authors and/or other copyright owners. Terms and conditions for use of this material are defined in the policies page.

\section{CLoK}

Central Lancashire online Knowledge www.clok.uclan.ac.uk

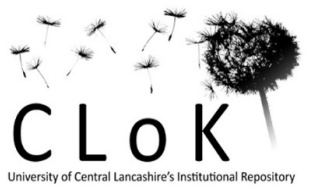




\section{Some results on dependent random variables and a connection with the multivariate s-increasing convex order}

\section{Milto Hadjikyriakou}

To cite this article: Milto Hadjikyriakou (2017): Some results on dependent random variables and a connection with the multivariate s-increasing convex order, Communications in Statistics - Theory and Methods, DOI: 10.1080/03610926.2017.1291977

To link to this article: http://dx.doi.org/10.1080/03610926.2017.1291977

Feb 2017.

Submit your article to this journal $\widetilde{ }$

Џ Article views: 10

Q View related articles $\sqsubset$

View Crossmark data $\asymp$ 


\title{
ACCEPTED MANUSCRIPT
}

\section{Some results on dependent random variables and a connection with the multivariate s-increasing convex order}

\author{
Milto Hadjikyriakou*
}

\begin{abstract}
In this paper some new concepts of dependence are introduced that generalize the concepts of positive and negative association. The new concepts of dependence are linked to the multivariate s-increasing convex order (Denuit and Mesfioui $(2010,2013)$ ). Furthermore, a Kolmogorov-type inequality and a Hàjek-Rènyi inequality are proven that lead to an asymptotic result for these new random objects.
\end{abstract}

Key words and phrases: positive association, negative association, Kolmogorov-type inequality, Hàjek-Rènyi inequality, multivariate s-increasing convex order.

AMS 2010 subject classification: 60E15, 60F15.

${ }^{*}$ School of Sciences, University of Central Lancashire, 12-14 University Avenue, Pyla, 7080 Larnaka, Cyprus (email: miltwh@gmail.com). 


\section{ACCEPTED MANUSCRIPT}

\section{Introduction}

Stochastic orders between random vectors have been studied extensively by various authors exactly because of their wide range of applications in several fields of probability and statistics. Some stochastic orders can be defined by reference to some class of measurable functions. Consider two $n$-dimensional random vectors $\boldsymbol{X}$ and $\boldsymbol{Y}$. The random vector $\boldsymbol{X}$ is said to be smaller than the random vector $\boldsymbol{Y}$ in the $\varsigma_{*}$ ordering associated to the class $\mathcal{U}_{*}^{[n]}$ of real-valued functions defined on (a subset of) the $n$-dimensional real space $\mathbb{R}^{n}$ if

$$
E[g(\boldsymbol{X})] \leq E[g(\boldsymbol{Y})] \text { for all } g \in \mathcal{U}_{*}^{[n]}
$$

Some of the most celebrated stochastic orders are the ones that involve convex functions. For example, in the case where $\mathcal{U}_{*}^{[1]}$ includes convex functions, (1) defines the univariate convex order while if $\mathcal{U}_{*}^{[n]}$ includes multivariate convex functions then the multivariate convex order is defined. The directionally convex order can be defined in a similar way. Multivariate extensions involving convex functions can also be found in Denuit et al (1999), Denuit and Mesfioui (2010, 2013) while recently Sordo (2016) introduced a multivariate extension of the increasing convex order that can be used in the case where the components of the random vectors that are involved are heterogeneous. For an interested reader we refer the books of Shaked and Shanthikumar (2007) and Müller and Stoyan (2002).

Let $\mathbf{s}=\left(s_{1}, s_{2}, \ldots, s_{n}\right)$ be a vector of non-negative integers and $S \subseteq \mathbb{R}^{n}$. Let $\mathcal{U}_{\mathbf{s}-i c x}(S)$ be the class of all functions $g: S \rightarrow \mathbb{R}$ such that

$$
\frac{\partial^{k_{1}+k_{2}+\cdots+k_{n}}}{\partial x_{1}^{k_{1}} \partial x_{2}^{k_{2}} \cdots \partial x_{n}^{k_{n}}} g\left(x_{1}, \ldots, x_{n}\right) \geq 0 \text { for all } x_{1}, \ldots, x_{n} \in S,
$$

where $k_{i}=0,1, \ldots, s_{i}, i=1,2, \ldots, n, k_{1}+k_{2}+\cdots+k_{n} \geq 1$.

Consider two $n$-dimensional random vectors $\left(X_{1}, \ldots, X_{n}\right)$ and $\left(Y_{1}, \ldots, Y_{n}\right)$ valued in $S$. Denuit and Mesfioui (2010) introduced the concept of the s-increasing convex order as follows:

\section{ACCEPTED MANUSCRIPT}




\section{ACCEPTED MANUSCRIPT}

Definition 1 The random vector $\left(X_{1}, \ldots, X_{n}\right)$ is said to be smaller than $\left(Y_{1}, \ldots, Y_{n}\right)$ in the $\mathbf{s}$ increasing convex order, which is denoted by $\left(X_{1}, \ldots, X_{n}\right) \leq_{s-i c x}\left(Y_{1}, \ldots, Y_{n}\right)$ if

$$
E\left[g\left(X_{1}, \ldots, X_{n}\right)\right] \leq E\left[g\left(Y_{1}, \ldots, Y_{n}\right)\right] \text { for all } g \in \mathcal{U}_{s-i c x}(S),
$$

provided that the expectations exist.

For this new stochastic order Denuit and Mesfioui (2010) provided the following result (Property $6.1(\mathrm{i}))$.

Lemma 2 Let $\left(X_{1}, \ldots, X_{n}\right)$ and $\left(Y_{1}, \ldots, Y_{n}\right)$ be nonnegative random variables. Then

$$
\mathbf{X} \leq_{s-i c x} \mathbf{Y} \Rightarrow \Psi(\mathbf{X}) \leq_{\left(\sum_{i=1}^{n} s_{i}\right)-i c x} \Psi(\mathbf{Y})
$$

for any non-negative function $\Psi$ in $\mathcal{U}_{s-i c x}$. In particular,

$$
\mathbf{X} \unlhd_{s-i c x} \mathbf{Y} \Rightarrow \sum_{i=1}^{n} \alpha_{i} X_{i} \unlhd_{\left(\sum_{i=1}^{n} s_{i}\right)-i c x} \sum_{i=1}^{n} \alpha_{i} Y_{i} \text { for any } \alpha_{1}, \ldots, \alpha_{n} \geq 0 .
$$

Denuit and Mesfioui (2013) generalized the above comparison for vectors of partial sums.

Lemma 3 If $\left(X_{1}, \ldots, X_{n}\right) \leq_{s-i c x}\left(Y_{1}, \ldots, Y_{n}\right)$ then

$$
\left(S_{1}, \ldots, S_{n}\right) \leq_{\left(s_{1}, s_{1}+s_{2}, \cdots, s_{1}+s_{2}+\cdots+s_{n}\right)-i c x}\left(T_{1}, \ldots, T_{n}\right)
$$

where $S_{j}=X_{1}+\cdots+X_{j}$ and $T_{j}=Y_{1}+\cdots+Y_{j}$ for $j=1, \ldots, n$.

It is of interest to study whether the results of Denuit and Mesfioui $(2010,2013)$ are applicable to vectors of random variables that are somehow dependent. A variety of concepts of dependence appear in the literature. These concepts have generated wide interest since they have a variety of applications in many different fields such as multivariate statistical analysis, reliability theory, actuarial science etc. Two of the most celebrated notions of dependence are positive and negative association, introduced by Esary et al (1967) and Joag-Dev and Proschan (1983) respectively. Let 


\section{ACCEPTED MANUSCRIPT}

us recall the definitions of positive and negative association since these two concepts are of major importance for what follows in this paper.

Definition 4 A finite collection of random variables $X_{1}, \ldots, X_{n}$ is said to be (positively) associated if

$$
\operatorname{Cov}\left(f\left(X_{1}, \ldots, X_{n}\right), g\left(X_{1}, \ldots, X_{n}\right)\right) \geq 0
$$

for any two componentwise nondecreasing functions $f, g$ provided that the covariance is defined. An infinite collection is associated if every finite subcollection is associated.

Definition 5 A finite collection of random variables $X_{1}, \ldots, X_{n}$ is said to be negatively associated (NA) if

$$
\operatorname{Cov}\left(f\left(X_{i}, i \in A\right), g\left(X_{j}, j \in B\right)\right) \leq 0
$$

for any disjoint subsets $A$ and $B$ of $\{1,2, \ldots, n\}$ and for any two componentwise nondecreasing functions $f, g$ on $\mathbb{R}^{|A|}$ and $\mathbb{R}^{|B|}$ respectively, provided that the covariance is defined. An infinite collection is negatively associated if every finite subcollection is negatively associated.

The last decades the notions of positive and negative association have been studied extensively by many researchers and among the various results obtained for these concepts are several generalized notions of dependence. Newman and Wright (1982) introduced the concept of demimartingales in order to provide a much more general class than positively associated random variables. The definition of demimartingales is given below.

Definition 6 Let $\left\{S_{n}, n \geq 1\right\}$ be a collection of random variables defined on a probability space $(\Omega, \mathcal{A}, \mathcal{P})$. The sequence $\left\{S_{n}, n \geq 1\right\}$ is called a demimartingale if for every componentwise nondecreasing function $f$ and for $j>i$

$$
E\left[\left(S_{j}-S_{i}\right) f\left(S_{1}, \ldots, S_{i}\right)\right] \geq 0
$$




\section{ACCEPTED MANUSCRIPT}

If moreover (3) is valid for any nonnegative componentwise nondecreasing function $f$, then $\left\{S_{n}, n \geq\right.$ 1) is called a demisubmartingale.

Note that it is straight forward to prove that the partial sums of mean zero positively associated random variables form a sequence of demimartingales. Motivated by the definition of demimartingales Christofides (2003) introduced the concept of N-demimartingales. It can easily be proven that the partial sums of mean zero negatively associated random variables satisfy the structure of an $\mathrm{N}$-demimartingale sequence.

Definition 7 Let $\left\{S_{n}, n \geq 1\right\}$ be a collection of random variables defined on a probability space $(\Omega, \mathcal{A}, \mathcal{P})$. The sequence $\left\{S_{n}, n \geq 1\right\}$ is called an $N$-demimartingale if for every componentwise nondecreasing function $f$ and for $j>i$

$$
E\left[\left(S_{j}-S_{i}\right) f\left(S_{1}, \ldots, S_{i}\right)\right] \leq 0
$$

If moreover (4) is valid for any nonnegative componentwise nondecreasing function $f$, then $\left\{S_{n}, n \geq\right.$ 1) is called a $\mathrm{N}$-demisupermartingale.

Results related to demimartingales and $\mathrm{N}$-demimartingales can be found in the monograph of Prakasa Rao (2012).

In this paper we provide the definitions of two new concepts of dependence similar in structure as the concepts of demimartingales and N-demimartingales. Firstly, we introduce the concept of a sequence that is said to have strong $\mathrm{N}$-demimartingale differences.

Definition 8 Let $\left\{S_{n}, n \in \mathbb{N}\right\}$ be a sequence of random variables with $S_{0} \equiv 0$. If for any $f$ and $g$ componentwise nondecreasing functions

$$
\operatorname{Cov}\left[g\left(S_{n+1}-S_{n}\right), f\left(S_{1}, S_{2}-S_{1}, \ldots, S_{n}-S_{n-1}\right)\right] \leq 0 \text { for } n=1,2, \ldots
$$

the sequence $\left\{S_{n}, n \in \mathbb{N}\right\}$ is said to have strong $N$-demimartingale differences. 


\section{ACCEPTED MANUSCRIPT}

The above definition is closely related to the concept of negative association. Let $\left\{X_{n}, n \in \mathbb{N}\right\}$ be a sequence of NA random variables and let $\left\{S_{n}, n \in \mathbb{N}\right\}$ be the sequence of their partial sums, i.e. $S_{n}=\sum_{i=1}^{n} X_{i}$, for $n \in \mathbb{N}$. Because of the negative association property we have that

$$
\operatorname{Cov}\left[g\left(S_{j+1}-S_{j}\right), f\left(S_{1}, S_{2}-S_{1}, \ldots, S_{j}-S_{j-1}\right)\right]=\operatorname{Cov}\left[g\left(X_{j+1}\right), f\left(X_{1}, X_{2}, \ldots, X_{j}\right)\right] \leq 0 .
$$

This latter inequality proves that the sequence of partial sums of NA random variables has strong $\mathrm{N}$-demimartingale differences.

Furthermore, it is trivial to verify that the random variables $X_{1}$ and $X_{2}$ are NA if and only if $X_{1}$ and $X_{1}+X_{2}$ have strong $\mathrm{N}$-demimartingale differences.

However, it is crucial to verify that the class of random variables that have the property of strong N-demimartingale differences does not include only the sequence of partial sums of negatively associated random variables but it is actually a wider class of random variables. This is proven via the counterexample that follows.

Example 9 Let $X_{1}, X_{2}, X_{3}$ and $X_{4}$ be random variables with joint probability mass function $P$ defined as

$$
\begin{gathered}
P(0,0,0,0)=\frac{1}{96}, P(1,0,0,0)=\frac{1}{48}, P(0,0,0,1)=\frac{7}{96}, P(1,0,0,1)=\frac{17}{144} \\
P(0,0,1,0)=\frac{1}{32}, P(1,0,1,0)=\frac{3}{144}, P(0,0,1,1)=\frac{5}{96}, P(1,0,1,1)=\frac{1}{144} \\
P(0,1,0,0)=\frac{1}{16}, P(1,1,0,0)=\frac{1}{36}, P(0,1,0,1)=\frac{35}{144}, P(1,1,0,1)=\frac{1}{9} \\
P(0,1,1,0)=\frac{1}{16}, P(1,1,1,0)=\frac{1}{72}, P(0,1,1,1)=\frac{19}{144}, P(1,1,1,1)=\frac{1}{72}
\end{gathered}
$$

where $P(a, b, c, d)=P\left(X_{1}=a, X_{2}=b, X_{3}=c, X_{4}=d\right)$ and let $S_{n}=\sum_{i=1}^{n} X_{i}$ for $n=1,2,3,4$.

It can be proven that $\left\{S_{1}, S_{2}, S_{3}, S_{4}\right\}$ satisfy (5) since for any $f$ and $g$ componentwise nondecreasing functions the following relations are valid

$$
\operatorname{Cov}\left(g\left(X_{2}\right), f\left(X_{1}\right)\right) \leq 0
$$




\section{ACCEPTED MANUSCRIPT}

$$
\operatorname{Cov}\left(g\left(X_{3}\right), f\left(X_{1}, X_{2}\right)\right) \leq 0,
$$

and

$$
\operatorname{Cov}\left(g\left(X_{4}\right), f\left(X_{1}, X_{2}, X_{3}\right)\right) \leq 0
$$

It can also be proven that for

$$
g(1,1)=4, g(0,1)=2, g(1,0)=1, g(0,0)=0
$$

and

$$
f(0,0)=1, f(0,1)=2, f(1,0)=1, f(1,1)=2 .
$$

the covariance

$$
\begin{aligned}
\operatorname{Cov}\left(f\left(X_{1}, X_{2}\right), g\left(X_{3}, X_{4}\right)\right) & =\frac{1}{1728}(79 f(0,0)+99 f(0,1)-95 f(1,0)-83 f(1,1)) \\
& =\frac{1}{108}>0 .
\end{aligned}
$$

The latter inequality proves that $\left\{X_{1}, X_{2}, X_{3}, X_{4}\right\}$ are not negatively associated.

Similar to the concept of sequences having strong N-demimartingale differences we can provide the definition of a sequence that is said to have strong demimartingale differences.

Definition 10 Let $\left\{S_{n}, n \in \mathbb{N}\right\}$ be a sequence of random variables with $S_{0} \equiv 0$. If for any $f$ and $g$ componentwise nondecreasing functions

$$
\operatorname{Cov}\left[g\left(S_{n+1}-S_{n}\right), f\left(S_{1}, S_{2}-S_{1}, \ldots, S_{n}-S_{n-1}\right)\right] \geq 0 \text { for } n=1,2, \ldots
$$

the sequence $\left\{S_{n}, n \in \mathbb{N}\right\}$ is said to have strong demimartingale differences.

Let $\left\{X_{n}, n \in \mathbb{N}\right\}$ be a sequence of associated random variables and let $\left\{S_{n}, n \in \mathbb{N}\right\}$ be the sequence of their partial sums, i.e. $S_{n}=\sum_{i=1}^{n} X_{i}$, for $n \in \mathbb{N}$. Then

$$
\operatorname{Cov}\left[g\left(S_{j+1}-S_{j}\right), f\left(S_{1}, S_{2}-S_{1}, \ldots, S_{j}-S_{j-1}\right)\right]=\operatorname{Cov}\left[g\left(X_{j+1}\right), f\left(X_{1}, X_{2}, \ldots, X_{j}\right)\right] \geq 0
$$




\section{ACCEPTED MANUSCRIPT}

proving that the sequence of partial sums of associated random variables satisfies (9).

Next we provide an example of a sequence that is said to have strong $\mathrm{N}$-demimartingale differences.

Example 11 Let $X_{1}, X_{2}, \ldots$ be normally distributed random variables with mean $\mu_{i}$ and variance $\sigma_{i}^{2}$ for $i=1,2, \ldots$ and let $X_{0} \equiv 0$. Define $Y_{i}=X_{i}-X_{i-1}$ for $i=1,2, \ldots$. Then $Y_{i}$ are normally distributed with mean $\mu_{i}-\mu_{i-1}$ and variance $\sigma_{i-1}^{2}+\sigma_{i}^{2}-2 \rho_{i-1, i} \sigma_{i-1} \sigma_{i}$ where $\rho_{i-1, i}=\operatorname{Corr}\left(X_{i-1}, X_{i}\right)$. Suppose that the bivariate distribution of the vector $\left(Y_{i}, Y_{j}\right)$ is given by the Farlie-Gumbel-Morgenstern system

$$
F_{Y_{i}, Y_{j}}(x, y)=F_{Y_{i}}(x) F_{Y_{j}}(y)\left\{1+\alpha_{i j}\left[1-F_{Y_{i}}(x)\right]\left[1-F_{Y_{j}}(y)\right]\right\}, \alpha_{i j} \in(-1,0)
$$

where $F_{Y_{i}}$ and $F_{Y_{j}}$ are the marginal cumulative distribution functions of the random variables $Y_{i}$ and $Y_{j}$. Schucany et al. (1978) proved that for the bivariate distribution described by (10)

$$
\operatorname{Cov}\left(Y_{i}, Y_{j}\right)=\frac{\alpha_{i j}}{\pi}
$$

and since $\alpha_{i j} \in(-1,0)$ the random variables $Y_{i}, Y_{j}$ are negatively correlated. But negatively correlated random variables that are normally distributed form a sequence of negatively associated random variables. Therefore for any $f$ and $g$ componentwise nondecreasing functions

$$
\operatorname{Cov}\left(g\left(X_{i+1}-X_{i}\right), f\left(X_{1}, X_{2}-X_{1}, \ldots, X_{i}-X_{i-1}\right)\right) \leq 0 \text { for } i=1,2, \ldots
$$

proving that $\left\{X_{n}, n \in \mathbb{N}\right\}$ forms a sequence that is said to have strong $N$-demimartingale differences.

In the case where the random variables $X_{i}$ 's are positively correlated then it is known that they are also positively associated. In this example it has been proven that it is also a sequence that is said to have strong $\mathrm{N}$-demimartingale differences.

Remark 12 Note that if the details of the example presented above are properly modified, i.e. $\alpha_{i j} \in(0,1)$, we can obtain an example of a sequence that is said to have strong demimartingale sequences. 


\section{ACCEPTED MANUSCRIPT}

Remark 13 The counterexample provided in Example 9 highlights the fact that a sequence of partial sums of random variables can satisfy the definition of a sequence that is said to have strong $N$-demimartingale differences without the random variables to be negatively associated. Furthermore, Example 11 provides a sequence of random variables that is said to have strong $N$-demimartingale differences even in the case where the random variables are positively associated. Additionally, the random variables in Example 11 can be negatively associated and at the same time the random variables themselves to be a sequence that is said to have strong $N$ demimartingale differences and not their partial sums. These two examples prove that the classes introduced in this paper are wider classes than the classes of positively and negatively associated random variables.

The purpose of this paper is to prove that a vector of random variables that are said to have strong $\mathrm{N}$-demimartingale differences (strong demimartingale differences) is smaller (larger) than the vector of their independent duplicates with respect to the multivariate s-increasing convex order. As a direct consequence this kind of stochastic ordering can also be obtained for negatively/positively associated random variables as well. The desired result is obtained as a direct application of a much more general comparison inequality proven in Section 2. Furthermore in Section 3, the comparison inequality is utilized to provide useful probability inequalities such as a Kolmogorov-type inequality, a Hàjek-Rènyi type inequality and a strong law for random variables that are said to have strong N-demimartingale differences.

\section{Comparison inequalities for moments of functions of random variables}

The two results that follow are instrumental for the proof of the main result of this section. For the details of their proofs the interested reader can study Theorem B (page 5) and Theorem A (page 9) 


\section{ACCEPTED MANUSCRIPT}

of Roberts and Varberg (1973) respectively.

Theorem 14 If $f: I \rightarrow \mathbb{R}$ is convex, then the left and right derivatives, denoted by $f_{-}^{\prime}(x)$ and $f_{+}^{\prime}(x)$ respectively, exist and are increasing.

Theorem 15 A function $f:(a, b) \rightarrow \mathbb{R}$ is convex if and only if there is an increasing function $g:(a, b) \rightarrow \mathbb{R}$ and a point $c \in(a, b)$ such that for all $x \in(a, b)$,

$$
f(x)-f(c)=\int_{c}^{x} g(t) d t
$$

Remark 16 Recall that a function $f: \mathbb{R}^{n} \rightarrow \mathbb{R}$ is considered to be a componentwise convex function if it is convex in each variable. Assume that $g: \mathbb{R}^{n} \rightarrow \mathbb{R}$ is a componentwise convex function. The function $f(x)=g\left(x_{1}, x_{2}, \ldots, x_{i-1}, x, x_{i+1}, \ldots, x_{n}\right)$ is a convex function with respect to $x$ for all $i=1,2, \ldots, n$ and therefore Theorems 14 and 15 are valid for the function $f$.

The theorem that follows provides a comparison theorem for the expectations of functions of random vectors that have strong N-demimartingale differences and their independent duplicates.

Theorem 17 Let $\left\{S_{n}, n \in \mathbb{N}\right\}$ be a sequence of random variables that have strong $N$-demimartingale differences and let $X_{i}=S_{i}-S_{i-1}$. Let $X_{i}^{*}$ be independent random variables such that $X_{i}={ }_{s t} X_{i}^{*}$. Then

$$
E\left[g\left(X_{1}, \ldots, X_{n}\right)\right] \leq E\left[g\left(X_{1}^{*}, \ldots, X_{n}^{*}\right)\right]
$$

for every componentwise convex function $g$ such that its right derivative with respect to the $i$-th component, is componentwise nondecreasing for all $i=1,2, \ldots, n$.

Proof. The proof is inspired by Christofides and Vaggelatou (2004). Without loss of generality we assume that $X_{1}, \ldots, X_{n}$ and $X_{1}^{*}, \ldots, X_{n}^{*}$ are independent. First, it needs to be proven that

$$
E\left[g\left(X_{1}, \ldots, X_{n-1}, X_{n}\right)\right] \leq E\left[g\left(X_{1}, \ldots, X_{n-1}, X_{n}^{*}\right)\right]
$$




\section{ACCEPTED MANUSCRIPT}

For any $x, x_{0} \in \mathbb{R}$ and for every componentwise convex function $g$ we can write

$$
\begin{aligned}
g\left(X_{1}, \ldots, X_{n-1}, x\right) & =g\left(X_{1}, \ldots, X_{n-1}, x_{0}\right)+\int_{x_{0}}^{x} h_{n}\left(X_{1}, \ldots, X_{n-1}, t\right) d t \\
& =g\left(X_{1}, \ldots, X_{n-1}, x_{0}\right)+\int_{\mathbb{R}}\left[I[x>t]-I\left[x_{0}>t\right]\right] h_{n}\left(X_{1}, \ldots, X_{n-1}, t\right) d t
\end{aligned}
$$

where $h_{n}$ is the right derivative of $g$ with respect to the last variable. Since the equality stated above is valid for all real numbers, it follows that

$$
g\left(X_{1}, \ldots, X_{n-1}, X_{n}\right)-g\left(X_{1}, \ldots, X_{n-1}, X_{n}^{*}\right)=\int_{\mathbb{R}}\left[I\left[X_{n}>t\right]-I\left[X_{n}^{*}>t\right]\right] h_{n}\left(X_{1}, \ldots, X_{n-1}, t\right) d t
$$

By taking expectations on both sides we have that

$$
\begin{aligned}
E[L H S] & =\int_{\mathbb{R}} E\left\{\left(I\left[X_{n}>t\right]-I\left[X_{n}^{*}>t\right]\right) h_{n}\left(X_{1}, \ldots, X_{n-1}, t\right)\right\} d t \\
& =\int_{\mathbb{R}}\left\{E\left[I\left[X_{n}>t\right] h_{n}\left(X_{1}, \ldots, X_{n-1}, t\right)\right]-E\left[I\left[X_{n}^{*}>t\right] h_{n}\left(X_{1}, \ldots, X_{n-1}, t\right)\right]\right\} d t \\
& =\int_{\mathbb{R}}\left\{E\left[I\left[X_{n}>t\right] h_{n}\left(X_{1}, \ldots, X_{n-1}, t\right)\right]-E\left(I\left[X_{n}^{*}>t\right]\right) E\left(h_{n}\left(X_{1}, \ldots, X_{n-1}, t\right)\right)\right\} d t \\
& =\int_{\mathbb{R}}\left\{E\left[I\left[X_{n}>t\right] h_{n}\left(X_{1}, \ldots, X_{n-1}, t\right)\right]-E\left(I\left[X_{n}>t\right]\right) E\left(h_{n}\left(X_{1}, \ldots, X_{n-1}, t\right)\right)\right\} d t \\
& =\int_{\mathbb{R}} \operatorname{Cov}\left(I\left(X_{n}>t\right), h_{n}\left(X_{1}, \ldots, X_{n-1}, t\right)\right) d t \\
& =\int_{\mathbb{R}} \operatorname{Cov}\left(I\left(S_{n}-S_{n-1}>t\right), h_{n}\left(S_{1}, S_{2}-S_{1} \ldots, S_{n-1}-S_{n-2}, t\right)\right) d t
\end{aligned}
$$

where the third equality follows by the independence of $X_{n}^{*}$ and $X_{1}, \ldots, X_{n-1}$ and the fourth by the fact that $X_{n}={ }_{s t} X_{n}^{*}$.

Observe that for all values of $t, I\left(S_{n}-S_{n-1}>t\right)$ is a nondecreasing function of $S_{n}-S_{n-1}$ and by assumption $h_{n}\left(S_{1}, \ldots, S_{n-1}-S_{n-2}, t\right)$ is a componentwise nondecreasing function of $S_{1}, S_{2}-$ $S_{1}, \ldots, S_{n-1}-S_{n-2}$. Therefore by the definition of $\left\{S_{n}, n \in \mathbb{N}\right\}$

$$
\operatorname{Cov}\left(I\left(S_{n}-S_{n-1}>t\right), h_{n}\left(S_{1}, \ldots, S_{n-1}-S_{n-2}, t\right)\right) \leq 0
$$




\section{ACCEPTED MANUSCRIPT}

This last inequality yields (12).

The proof of (11) follows by induction. For $n=2$ we have that

$$
E\left[g\left(X_{1}^{*}, X_{2}^{*}\right)\right]=E\left[g\left(X_{1}, X_{2}^{*}\right)\right] \geq E\left[g\left(X_{1}, X_{2}\right)\right] \text { because of (12). }
$$

Next, assume that the statement is true for $n-1$. Then for $n$-dimensional random vectors we have that

$$
\begin{aligned}
E\left[g\left(X_{1}^{*}, \ldots, X_{n}^{*}\right)\right] & =E\left\{E\left[g\left(X_{1}^{*}, \ldots, X_{n}^{*}\right) \mid X_{n}^{*}\right]\right\} \\
& =\int_{\mathbb{R}} E\left[g\left(X_{1}^{*}, \ldots, X_{n}^{*}\right) \mid X_{n}^{*}=x\right] d F_{X_{n}^{*}}(x) \\
& =\int_{\mathbb{R}} E\left[g\left(X_{1}^{*}, \ldots, X_{n-1}^{*}, x\right)\right] d F_{X_{n}^{*}}(x) \\
& \geq \int_{\mathbb{R}} E\left[g\left(X_{1}, \ldots, X_{n-1}, x\right)\right] d F_{X_{n}^{*}}(x) \text { (by the induction hypothesis) } \\
& =\int_{\mathbb{R}} E\left[g\left(X_{1}, \ldots, X_{n-1}, X_{n}^{*}\right) \mid X_{n}^{*}=x\right] d F_{X_{n}^{*}}(x) \\
& =E\left\{E\left[g\left(X_{1}, \ldots, X_{n-1}, X_{n}^{*}\right) \mid X_{n}^{*}\right]\right\} \\
& =E\left[g\left(X_{1}, \ldots, X_{n-1}, X_{n}^{*}\right)\right] \\
& \geq E\left[g\left(X_{1}, \ldots, X_{n-1}, X_{n}\right)\right]
\end{aligned}
$$

where the fourth equality follows from the independence of $X_{n}^{*}$ and $X_{1}, \ldots, X_{n-1}$ and the last equality follows by (12).

Next, we provide the comparison inequality for a sequence of random variables that is said to have strong demimartingale differences. The proof follows by applying the same steps as in the proof of Theorem 17 and therefore is omitted for brevity.

Theorem 18 Let $\left\{S_{n}, n \in \mathbb{N}\right\}$ be a sequence of random variables that have strong demimartingale differences and let $X_{i}=S_{i}-S_{i-1}$. Let $X_{i}^{*}$ be independent random variables such that $X_{i}={ }_{s t} X_{i}^{*}$. Then

$$
E\left[g\left(X_{1}, \ldots, X_{n}\right)\right] \geq E\left[g\left(X_{1}^{*}, \ldots, X_{n}^{*}\right)\right]
$$




\section{ACCEPTED MANUSCRIPT}

for every componentwise convex function $g$ such that its right derivative with respect to the $i$-th component is componentwise nondecreasing for all $i=1,2, \ldots, n$.

\section{A connection to the multivariate $s$-increasing convex order}

The results provided in Theorems 17 and 18 allow us to give an answer to the question stated earlier i.e. whether s-increasing convex order can be obtained for random variables that are somehow dependent.

Henceforth, following the notation of Denuit and Mesfioui (2013), for all vectors $\mathbf{S}=\left(s_{1}, s_{2}, \ldots, s_{n}\right)$ of non-negative integers define

$$
\Sigma_{i}(\mathbf{s})=s_{1}+s_{2}+\cdots+s_{i}, \quad i=1,2, \ldots, n
$$

and

$$
\Sigma(\mathbf{s})=\left(\Sigma_{1}(\mathbf{s}), \ldots, \Sigma_{n}(\mathbf{s})\right)
$$

Theorem 19 Let $\left\{S_{n}, n \in \mathbb{N}\right\},\left\{X_{n}, n \in \mathbb{N}\right\}$ and $\left\{X_{n}^{*}, n \in \mathbb{N}\right\}$ be as stated in Theorem 17 and let $\widehat{S}_{n}=\sum_{i=1}^{n} X_{i}^{*}$. Then for all vectors $\boldsymbol{s}=\left(s_{1}, s_{2}, \ldots, s_{n}\right)$ where $s_{i} \geq 2$ for all $i=1,2, \ldots, n$

1 .

$$
\left(X_{1}, \ldots, X_{n}\right) \unlhd_{s-i c x}\left(X_{1}^{*}, \ldots, X_{n}^{*}\right)
$$

2 .

$$
\left(X_{1}, \ldots, X_{k}\right) \unlhd_{s-i c x}\left(X_{1}^{*}, \ldots, X_{k}^{*}\right) \text { for } k=1,2, \ldots, n
$$

3. In the case of nonnegative random variables,

$$
S_{k} \unlhd_{\sum_{i=1}^{k} s_{i}-i c x} \widehat{S}_{k} \text { for } k=1,2, \ldots, n,
$$

4.

$$
\left(S_{1}, \ldots, S_{n}\right) \leq_{\Sigma(s)-i c x}\left(\widehat{S}_{1}, \ldots, \widehat{S}_{n}\right) .
$$




\section{ACCEPTED MANUSCRIPT}

Proof. Note that Theorem 17 was proven for any componentwise convex function $g$ such that its right derivative with respect to the $i$-th component is componentwise nondecreasing for all $i=1,2, \ldots, n$. It is essential to mention that the result is valid for functions that are not necessarily differentiable. In the case where the function $g$ satisfies condition (2) for $s_{i} \geq 2$, then it is considered to be componentwise convex and its derivatives are componentwise nondecreasing functions. Therefore, the assumptions of Theorem 17 are satisfied and the proof of (14) follows directly from Theorem 17.

The fact that the s-icx order is closed under marginalization leads to (15). The validity of (16) follows by Lemma 2 (Property (6.1i) of Denuit and Mesfioui (2010)). Finally, (17) follows by applying the result of Lemma 2 (Proposition (3.1) of Denuit and Mesfioui (2013)).

In the case of sequences that are said to have strong demimartingale differences a similar theorem can be obtained with the reversed inequalities.

Theorem 20 Let $\left\{S_{n}, n \in \mathbb{N}\right\},\left\{X_{n}, n \in \mathbb{N}\right\}$ and $\left\{X_{n}^{*}, n \in \mathbb{N}\right\}$ be as stated in Theorem 18 and let $\widehat{S}_{n}=\sum_{i=1}^{n} X_{i}^{*}$. Then for all vectors $\boldsymbol{s}=\left(s_{1}, s_{2}, \ldots, s_{n}\right)$ where $s_{i} \geq 2$ for all $i=1,2, \ldots, n$

1.

$$
\left(X_{1}, \ldots, X_{n}\right) \geq_{s-i c x}\left(X_{1}^{*}, \ldots, X_{n}^{*}\right)
$$

2.

$$
\left(X_{1}, \ldots, X_{k}\right) \geq_{s-i c x}\left(X_{1}^{*}, \ldots, X_{k}^{*}\right) \text { for } k=1,2, \ldots, n,
$$

3. In the case of nonnegative random variables,

$$
S_{k} \geq_{\sum_{i=1}^{k} s_{i}-i c x} \widehat{S}_{k} \text { for } k=1,2, \ldots, n,
$$

4.

$$
\left(S_{1}, \ldots, S_{n}\right) \geq_{\Sigma(s)-i c x}\left(\widehat{S}_{1}, \ldots, \widehat{S}_{n}\right)
$$




\section{ACCEPTED MANUSCRIPT}

Remark 21 It is worth mentioning that the results of Theorems 19 and 20 can lead to similar inequalities for negatively and positively associated random variables respectively.

\section{Some results on dependent random variables}

Even though the motivation for this research work was to provide a connection between the new concepts of dependence and the multivariate s-increasing convex order, the comparison inequality that was proven in Section 2, allows us to obtain some very useful probability inequalities. Firstly, let's state as a remark an observation that is instrumental for the rest of the paper.

Remark 22 Theorem 17 is valid for every function $g$ that is componentwise convex and its right derivative with respect to the any of its component is a componentwise nondecreasing function.

The function

$$
f_{1}\left(x_{1}, x_{2}, \ldots, x_{n}\right)=\sum_{i=1}^{n} x_{i}
$$

satisfies both conditions.

Two useful functions that also posses the desired properties (see the Appendix for the proofs) are

$$
f_{2}\left(x_{1}, x_{2}, \ldots, x_{n}\right)=\max _{1 \leq k \leq n} \sum_{i=1}^{k} x_{i}
$$

and

$$
f_{3}\left(x_{1}, x_{2}, \ldots, x_{n}\right)=\max _{1 \leq k \leq n}\left(\sum_{i=1}^{k} x_{i}\right)^{2} \text {. }
$$

A direct consequence of Theorem 17 and of the remark stated above is the result that follows.

Theorem 23 Let $\left\{S_{n}, n \in \mathbb{N}\right\}$ be a sequence of random variables that have strong $N$-demimartingale differences and let $X_{i}=S_{i}-S_{i-1}$. Let $X_{i}^{*}$ be independent random variables such that $X_{i}=_{s t} X_{i}^{*}$. Then

$$
E\left(f\left(\sum_{i=1}^{n} X_{i}\right)\right) \leq E\left(f\left(\sum_{i=1}^{n} X_{i}^{*}\right)\right)
$$




\section{ACCEPTED MANUSCRIPT}

and

$$
E\left(f\left(\max _{1 \leq k \leq n} \sum_{i=1}^{k} X_{i}\right)\right) \leq E\left(f\left(\max _{1 \leq k \leq n} \sum_{i=1}^{k} X_{i}^{*}\right)\right)
$$

for every $f$ increasing convex function.

Proof. By using Remark 22 and the fact that $f$ is an increasing convex function, the functions $g_{1}\left(x_{1}, \ldots, x_{n}\right)=f\left(\sum_{i=1}^{n} x_{i}\right)$ and $g_{2}\left(x_{1}, \ldots, x_{n}\right)=f\left(\max _{1 \leq k \leq n} \sum_{i=1}^{k} x_{i}\right)$ satisfy the conditions of Theorem 17 and therefore inequalities (18) and (19) follow directly from (11).

The fact that the sequence of partial sums of NA random variables satisfies (5) leads to the corollaries that follow.

Corollary 24 Let $\left\{X_{n}, n \in \mathbb{N}\right\}$ be a sequence of NA random variables and let $X_{i}^{*}$ be independent random variables such that $X_{i}=_{s t} X_{i}^{*}$ for $i=1,2, \ldots$ Then

$$
E\left[g\left(X_{1}, \ldots, X_{n}\right)\right] \leq E\left[g\left(X_{1}^{*}, \ldots, X_{n}^{*}\right)\right]
$$

for every componentwise convex function $g$ such that its right derivative with respect to the $i$-th component is componentwise nondecreasing for all $i=1,2, \ldots, n$.

Corollary 25 Let $\left\{X_{n}, n \in \mathbb{N}\right\}$ be a sequence of $N A$ random variables and let $X_{i}^{*}$ be independent random variables such that $X_{i}={ }_{s t} X_{i}^{*}$ for $i=1,2, \ldots$ Then

$$
E\left(f\left(\sum_{i=1}^{n} X_{i}\right)\right) \leq E\left(f\left(\sum_{i=1}^{n} X_{i}^{*}\right)\right)
$$

and

$$
E\left(f\left(\max _{1 \leq k \leq n} \sum_{i=1}^{k} X_{i}\right)\right) \leq E\left(f\left(\max _{1 \leq k \leq n} \sum_{i=1}^{k} X_{i}^{*}\right)\right)
$$

for every $f$ increasing convex function.

Remark 26 Observe that (21) is in full agreement with inequality (1.3) presented in Shao (2000).

Theorem 17 is instrumental for proving the Kolmogorov-type inequality that follows. 


\section{ACCEPTED MANUSCRIPT}

Corollary 27 Let $S_{n}, X_{n}$ and $X_{n}^{*}$ be as stated in Theorem 17 with $E\left(X_{i}\right)=0$ and $E X_{i}^{2}<\infty$ for all $i \in$ $\mathbb{N}$. Then for $\epsilon>0$

$$
P\left(\max _{1 \leq k \leq n}\left|S_{k}\right| \geq \epsilon\right) \leq \frac{4}{\epsilon^{2}} \sum_{i=1}^{n} E\left(X_{i}^{2}\right)
$$

Proof.

$$
\begin{aligned}
P\left(\max _{1 \leq k \leq n}\left|S_{k}\right| \geq \epsilon\right) & \leq P\left(\left(\max _{1 \leq k \leq n}\left|S_{k}\right|\right)^{2} \geq \epsilon^{2}\right) \\
& =P\left(\max _{1 \leq k \leq n} S_{k}^{2} \geq \epsilon^{2}\right) \\
& \leq \frac{1}{\epsilon^{2}} E\left(\max _{1 \leq k \leq n} S_{k}^{2}\right) \\
& \leq \frac{1}{\epsilon^{2}} E\left(\max _{1 \leq k \leq n}\left(\sum_{i=1}^{k} X_{i}^{*}\right)^{2}\right) \\
& \leq \frac{4}{\epsilon^{2}} \sum_{i=1}^{n} E\left(X_{i}^{*}\right)^{2} \\
& =\frac{4}{\epsilon^{2}} \sum_{i=1}^{n} E\left(X_{i}\right)^{2}
\end{aligned}
$$

where the third inequality follows by applying the result of Theorem 17 for the function $g\left(x_{1}, \ldots, x_{n}\right)=$ $\max _{1 \leq k \leq n}\left(x_{1}+\cdots+x_{k}\right)^{2}$ and the fourth inequality by Doob's inequality for martingales.

The Kolmogorov-type inequality is the key result for obtaining the Hàjek-Rènyi inequalities for random variables that are said to have strong $\mathrm{N}$-demimartingale differences. The proof can be obtained by applying standard arguments (see for example Chen et al.(1999)) and therefore is omitted for brevity.

Corollary 28 Let $S_{n}$ and $X_{n}$ be as stated in Theorem 17 with $E X_{i}^{2}<\infty$ for all $i \in \mathbb{N}$ and let $\left\{b_{n}, n \in \mathbb{N}\right\}$ be a nondecreasing sequence of positive real numbers. Then

$$
P\left(\max _{1 \leq k \leq n}\left|\frac{1}{b_{k}}\left(S_{k}-E S_{k}\right)\right| \geq \epsilon\right) \leq \frac{16}{\epsilon^{2}} \sum_{i=1}^{n} \frac{\operatorname{Var}\left(X_{i}\right)}{b_{i}^{2}}
$$

and

$$
P\left(\max _{m \leq k \leq n}\left|\frac{1}{b_{k}}\left(S_{k}-E S_{k}\right)\right| \geq \epsilon\right) \leq \frac{64}{\epsilon^{2}}\left(\sum_{i=m+1}^{n} \frac{\operatorname{Var}\left(X_{i}\right)}{b_{i}^{2}}+\sum_{i=1}^{n} \frac{\operatorname{Var}\left(X_{i}\right)}{b_{m}^{2}}\right)
$$




\section{ACCEPTED MANUSCRIPT}

As a direct consequence of the Hàjek-Rènyi inequality we can easily obtain the following strong law for random variables that are said to have strong $\mathrm{N}$-demimartingale differences. Again the proof can be obtained by applying similar steps as in the proof of Theorem 3.1 of Chen et al.(1999) and therefore is omitted.

Theorem 29 Let $S_{n}$ and $X_{n}$ be as stated in Theorem 17 and let $\left\{b_{n}, n \geq 1\right\}$ be a sequence of positive nondecreasing real numbers with $\sum_{n=1}^{\infty} \frac{\operatorname{Var} X_{n}}{b_{n}^{2}}<\infty$.

Then for any $0<r<2$,

$$
E \sup _{n}\left(\frac{\left|S_{n}\right|}{b_{n}}\right)^{r}<\infty .
$$

Assume that $0<b_{n} \uparrow \infty$, then for $n \rightarrow \infty$

$$
\frac{S_{n}-E S_{n}}{b_{n}} \rightarrow 0 \text { a.s.. }
$$

The result of the Theorem that follows, derives directly by applying the comparison inequality obtained in Theorem 18.

Theorem 30 Let $\left\{S_{n}, n \in \mathbb{N}\right\}$ be a sequence of random variables that have strong demimartingale differences and let $X_{i}=S_{i}-S_{i-1}$. Let $X_{i}^{*}$ be independent random variables such that $X_{i}={ }_{s t} X_{i}^{*}$. Then

$$
E\left(f\left(\sum_{i=1}^{n} X_{i}\right)\right) \geq E\left(f\left(\sum_{i=1}^{n} X_{i}^{*}\right)\right)
$$

and

$$
E\left(f\left(\max _{1 \leq k \leq n} \sum_{i=1}^{k} X_{i}\right)\right) \geq E\left(f\left(\max _{1 \leq k \leq n} \sum_{i=1}^{k} X_{i}^{*}\right)\right)
$$

for every $f$ increasing convex function.

Remark 31 Denuit et al. (2001) introduced another notion of dependence that is very useful for actuaries, namely the positive cumulative dependence (PCD). Based on Theorem 3.1 of their paper, the result stated in (25) is also valid for random variables that are PCD. Since the dependence notion proposed in this paper is stronger than PCD, (25) can be reduced to a known result. 


\section{ACCEPTED MANUSCRIPT}

Since the concept of positive association is closely related to the concept of sequences with strong demimartingale differences we can easily obtain the results that follow by applying the result of Theorem 18 .

Corollary 32 Let $\left\{X_{n}, n \in \mathbb{N}\right\}$ be a sequence of associated random variables and let $X_{i}^{*}$ be independent random variables such that $X_{i}=_{s t} X_{i}^{*}$ for $i=1,2, \ldots$ Then

$$
E\left[g\left(X_{1}, \ldots, X_{n}\right)\right] \geq E\left[g\left(X_{1}^{*}, \ldots, X_{n}^{*}\right)\right]
$$

for every componentwise convex function $g$ such that its right derivative with respect to the $i$-th component, $h_{i}$ is componentwise nondecreasing for all $i=1,2, \ldots, n$.

Corollary 33 Let $\left\{X_{n}, n \in \mathbb{N}\right\}$ be a sequence of associated random variables and let $X_{i}^{*}$ be independent random variables such that $X_{i}=_{s t} X_{i}^{*}$ for $i=1,2, \ldots$ Then

$$
E\left(f\left(\sum_{i=1}^{n} X_{i}\right)\right) \geq E\left(f\left(\sum_{i=1}^{n} X_{i}^{*}\right)\right)
$$

and

$$
E\left(f\left(\max _{1 \leq k \leq n} \sum_{i=1}^{k} X_{i}\right)\right) \geq E\left(f\left(\max _{1 \leq k \leq n} \sum_{i=1}^{k} X_{i}^{*}\right)\right)
$$

for every $f$ increasing convex function. 


\section{ACCEPTED MANUSCRIPT}

\section{References}

[1] Chen, P., Gan, S., Liu, J., (1999): The Hàjeck-Rènyi inequality for the NA random variables and its applicaton. Statist. Probab. Lett. 43, 99-105.

[2] Christofides, T.C. (2003): Maximal inequalities for N-demimartingales. Arch. Inequal. and Appl. 50, 397-408.

[3] Christofides, T.C, Vaggelatou, E. (2004): A connection between supermodular ordering and positive/negative association. Journal of Multivariate Analysis 88, 138-151.

[4] Denuit, M., Lefevre, C., and Mesfioui, M. H. (1999): A class of bivariate stochastic orderings, with applications in actuarial sciences. Insurance: Mathematics and Economics, 24, 31-50.

[5] Denuit, M., Dhaene, J., and Ribas, C. (2001): Does positive dependence between individual risks increase stop-loss premiums? Insurance: Mathematics and Economics, 28, 305-308.

[6] Denuit, M., Mesfioui, M. (2010): Generalized increasing convex and directionally convex orders.J. Appl. Prob. 47, 264-276.

[7] Denuit, M., Mesfioui, M. (2013): Ordering functions of random vectors, with application to partial sums.J. Theor. Prob. 26, 474-479.

[8] Esary, J., Proschan, F., Walkup, D., (1967): Association of random variables with applications. Ann. Math. Statist. 38, 1466-1474.

[9] Joag-Dev, K. and Proschan, F. (1983): Negative association of random variables with applications. Ann. Statist. 11, 286-295.

[10] Müller, A., and Stoyan, D. (2002): Comparison methods for stochastic models and risks, Wiley, New York. 


\section{ACCEPTED MANUSCRIPT}

[11] Newman, C.M. and A.L. Wright (1982): Associated random variables and martingale inequalities. Z. Wahrsch. Verw. Gebiete. 59, 361-371.

[12] Prakasa Rao, B.L.S., (2012). Associated Sequences, Demimartingales and Nonparametric Inference. Birkhuser, Basel.

[13] Roberts, A.W., Varberg, D.E. (1973): Convex Functions. Academic Press. New York.

[14] Schucany, W. R., Parr, W. C., and Boyer, J. E. (1978): Correlation structure in farlie-gumbelmorgenstern distributions. Biometrika, 65, 650-653.

[15] Shaked, M., and Shanthikumar, J. G. (2007): Stochastic orders. In: Series: Springer Series in Statistics, Springer.

[16] Shao, Q.M., (2000): A comparison theorem on moment inequalities between negatively associated and independent random variables. J. Theor. Probab. 13, 343-356.

[17] Sordo, M. A. (2016): A multivariate extension of the increasing convex order to compare risks. Insurance: Mathematics and Economics, 68, 224-230. 


\section{ACCEPTED MANUSCRIPT}

\section{Appendix}

Lemma 34 Consider the following functions

$$
f\left(x_{1}, \ldots, x_{n}\right)=\max \left\{x_{1}, x_{1}+x_{2}, \ldots, x_{1}+x_{2}+\cdots+x_{n}\right\}
$$

and

$$
g\left(x_{1}, \ldots, x_{n}\right)=\max \left\{x_{1}^{2},\left(x_{1}+x_{2}\right)^{2}, \ldots,\left(x_{1}+x_{2}+\cdots+x_{n}\right)^{2}\right\} .
$$

The right derivatives of these functions with respect to the $i$-th component are componentwise nondecreasing functions for all $i=1,2, \ldots, n$.

Proof. Without loss of generality we will calculate the right derivative of these functions with respect to their last variable.

Let $h_{1}(t)=f_{+}^{\prime}\left(x_{1}, x_{2}, \ldots, x_{n-1}, t\right)$ and $s_{j}=\sum_{i=1}^{j} x_{i}$. Then

$$
\begin{aligned}
h_{1}(t) & =\lim _{h \rightarrow 0^{+}} \frac{f\left(x_{1}, x_{2}, \ldots, x_{n-1}, t+h\right)-f\left(x_{1}, x_{2}, \ldots, x_{n-1}, t\right)}{h} \\
& =\lim _{h \rightarrow 0^{+}} \frac{\max \left\{s_{1}, s_{2}, \ldots, s_{n-1}, s_{n-1}+t+h\right\}-\max \left\{s_{1}, s_{2}, \ldots, s_{n-1}, s_{n-1}+t\right\}}{h} \\
& =\lim _{h \rightarrow 0^{+}} \frac{\max \left\{\max \left\{s_{1}, \ldots, s_{n-1}\right\}, s_{n-1}+t+h\right\}-\max \left\{\max \left\{s_{1}, \ldots, s_{n-1}\right\}, s_{n-1}+t\right\}}{h} .
\end{aligned}
$$

Let $\max \left\{s_{1}, s_{2}, \ldots, s_{n-1}\right\}=s_{j}, j=1,2, \ldots, n-1$. Then

$$
f_{+}^{\prime}\left(x_{1}, x_{2}, \ldots, x_{n-1}, t\right)=\lim _{h \rightarrow 0^{+}} \frac{\max \left\{s_{j}, s_{n-1}+t+h\right\}-\max \left\{s_{j}, s_{n-1}+t\right\}}{h} .
$$

$\underline{\text { Case } 1}$

Let $\max \left\{s_{j}, s_{n-1}+t\right\}=s_{j}$. The value for $h$ can be chosen sufficiently small such that $s_{j}>$ $s_{n-1}+t+h$. Therefore

$$
f_{+}^{\prime}\left(x_{1}, x_{2}, \ldots, x_{n-1}, t\right)=0
$$




\section{ACCEPTED MANUSCRIPT}

\section{Case 2}

Let $\max \left\{s_{j}, s_{n-1}+t\right\}=s_{n-1}+t$. Then $s_{n-1}+t+h>s_{j}$. Hence

$$
f_{+}^{\prime}\left(x_{1}, x_{2}, \ldots, x_{n-1}, t\right)=\lim _{h \rightarrow 0^{+}} \frac{\left(s_{n-1}+t+h\right)-\left(s_{n-1}+t\right)}{h}=1 .
$$

Thus, $f_{+}^{\prime}\left(x_{1}, x_{2}, \ldots, x_{n-1}, t\right)$ is a componentwise nondecreasing function.

For the second function we define $h_{2}(t)=g_{+}^{\prime}\left(x_{1}, x_{2}, \ldots, x_{n-1}, t\right)$. Then

$$
\begin{aligned}
h_{2}(t) & =\lim _{h \rightarrow 0^{+}} \frac{g\left(x_{1}, x_{2}, \ldots, x_{n-1}, t+h\right)-g\left(x_{1}, x_{2}, \ldots, x_{n-1}, t\right)}{h} \\
& =\lim _{h \rightarrow 0^{+}} \frac{\max \left\{s_{1}^{2}, s_{2}^{2}, \ldots, s_{n-1}^{2},\left(s_{n-1}+t+h\right)^{2}\right\}-\max \left\{s_{1}^{2}, s_{2}^{2}, \ldots, s_{n-1}^{2},\left(s_{n-1}+t\right)^{2}\right\}}{h} \\
& =\lim _{h \rightarrow 0^{+}} \frac{\max \left\{\max \left\{s_{1}^{2}, \ldots, s_{n-1}^{2}\right\},\left(s_{n-1}+t+h\right)^{2}\right\}-\max \left\{\max \left\{s_{1}^{2}, \ldots, s_{n-1}^{2}\right\},\left(s_{n-1}+t\right)^{2}\right\}}{h} .
\end{aligned}
$$

Let $\max \left\{s_{1}^{2}, \ldots, s_{n-1}^{2}\right\}=s_{j}^{2}$, for $j=1,2, \ldots, n-1$. Then

$$
g_{+}^{\prime}\left(x_{1}, x_{2}, \ldots, x_{n-1}, t\right)=\lim _{h \rightarrow 0^{+}} \frac{\max \left\{s_{j}^{2},\left(s_{n-1}+t+h\right)^{2}\right\}-\max \left\{s_{j}^{2},\left(s_{n-1}+t\right)^{2}\right\}}{h} .
$$

Note that

$$
\left(s_{n-1}+t\right)^{2}-s_{j}^{2}=\left(2 s_{j}+s+t\right)(s+t)
$$

and

$$
\left(s_{n-1}+t+h\right)^{2}-s_{j}^{2}=\left(2 s_{j}+s+t+h\right)(s+t+h),
$$

where $s=x_{j+1}+x_{j+2}+\cdots+x_{n-1}$. 


\section{ACCEPTED MANUSCRIPT}

\section{$\underline{\text { Case } 1}$}

Let $2 s_{j}+s+t>0$ and $s+t>0$. This leads to

$$
\max \left\{s_{j}^{2},\left(s_{n-1}+t\right)^{2}\right\}=\left(s_{n-1}+t\right)^{2}
$$

and since $2 s_{j}+s+t+h>0$ and $s+t+h>0$ we also have that

$$
\max \left\{s_{j}^{2},\left(s_{n-1}+t+h\right)^{2}\right\}=\left(s_{n-1}+t+h\right)^{2} .
$$

Hence

$$
\begin{aligned}
g_{+}^{\prime}\left(x_{1}, x_{2}, \ldots, x_{n-1}, t\right) & =\lim _{h \rightarrow 0^{+}} \frac{\left(s_{n-1}+t+h\right)^{2}-\left(s_{n-1}+t\right)^{2}}{h} \\
& =\lim _{h \rightarrow 0^{+}} \frac{h\left[2\left(s_{n-1}+t\right)+h\right]}{h} \\
& =2\left(s_{n-1}+t\right) .
\end{aligned}
$$

\section{$\underline{\text { Case } 2}$}

Let $2 s_{j}+s+t>0$ and $s+t<0$. This leads to

$$
\max \left\{s_{j}^{2},\left(s_{n-1}+t\right)^{2}\right\}=s_{j}^{2}
$$

The value of $h$ can be chosen sufficiently small such that $2 s_{j}+s+t+h>0$ and $s+t+h<0$. Then

$$
\max \left\{s_{j}^{2},\left(s_{n-1}+t+h\right)^{2}\right\}=s_{j}^{2}
$$

Hence

$$
g_{+}^{\prime}\left(x_{1}, x_{2}, \ldots, x_{n-1}, t\right)=\lim _{h \rightarrow 0^{+}} \frac{s_{j}^{2}-s_{j}^{2}}{h}=0
$$

$\underline{\text { Case } 3}$ 


\section{ACCEPTED MANUSCRIPT}

Let $2 s_{j}+s+t<0$ and $s+t>0$. This leads to

$$
\max \left\{s_{j}^{2},\left(s_{n-1}+t\right)^{2}\right\}=s_{j}^{2}
$$

The value of $h$ can be chosen sufficiently small such that $2 s_{j}+s+t+h<0$ and $s+t+h>0$. Then

$$
\max \left\{s_{j}^{2},\left(s_{n-1}+t+h\right)^{2}\right\}=s_{j}^{2}
$$

Hence

$$
g_{+}^{\prime}\left(x_{1}, x_{2}, \ldots, x_{n-1}, t\right)=\lim _{h \rightarrow 0^{+}} \frac{s_{j}^{2}-s_{j}^{2}}{h}=0
$$

$\underline{\text { Case } 4}$

Let $2 s_{j}+s+t<0$ and $s+t<0$. This leads to

$$
\max \left\{s_{j}^{2},\left(s_{n-1}+t\right)^{2}\right\}=\left(s_{n-1}+t\right)^{2} .
$$

The value of $h$ can be chosen sufficiently small such that $2 s_{j}+s+t+h<0$ and $s+t+h<0$. Then

$$
\max \left\{s_{j}^{2},\left(s_{n-1}+t+h\right)^{2}\right\}=\left(s_{n-1}+t+h\right)^{2} .
$$

Hence

$$
\begin{aligned}
g_{+}^{\prime}\left(x_{1}, x_{2}, \ldots, x_{n-1}, t\right) & =\lim _{h \rightarrow 0^{+}} \frac{\left(s_{n-1}+t+h\right)^{2}-\left(s_{n-1}+t\right)^{2}}{h} \\
& =\lim _{h \rightarrow 0^{+}} \frac{h\left[2\left(s_{n-1}+t\right)+h\right]}{h} \\
& =2\left(s_{n-1}+t\right) .
\end{aligned}
$$

Case 5 


\section{ACCEPTED MANUSCRIPT}

Let $s+t=0 \Rightarrow\left(s_{n-1}+t\right)^{2}=s_{j}^{2}$. Then $\left(s_{n-1}+t+h\right)^{2}-s_{j}^{2}=\left(2 s_{j}+h\right) h$.

(i) If $2 s_{j}+h>0$ then

$$
\max \left\{s_{j}^{2},\left(s_{n-1}+t+h\right)^{2}\right\}=\left(s_{n-1}+t+h\right)^{2} .
$$

Then

$$
\begin{aligned}
g_{+}^{\prime}\left(x_{1}, x_{2}, \ldots, x_{n-1}, t\right) & =\lim _{h \rightarrow 0^{+}} \frac{\left(s_{n-1}+t+h\right)^{2}-s_{j}^{2}}{h} \\
& =\lim _{h \rightarrow 0^{+}} \frac{\left(2 s_{j}+h\right) h}{h} \\
& =2 s_{j} .
\end{aligned}
$$

(ii) If $2 s_{j}+h \leq 0$ then

$$
\max \left\{s_{j}^{2},\left(s_{n-1}+t+h\right)^{2}\right\}=s_{j}^{2}
$$

Then

$$
g_{+}^{\prime}\left(x_{1}, x_{2}, \ldots, x_{n-1}, t\right)=\lim _{h \rightarrow 0^{+}} \frac{s_{j}^{2}-s_{j}^{2}}{h}=0
$$

$\underline{\text { Case } 6}$

Let $2 s_{j}+s+t=0 \Rightarrow\left(s_{n-1}+t\right)^{2}=s_{j}^{2}$. Then $\left(s_{n-1}+t+h\right)^{2}-s_{j}^{2}=(s+t+h) h$.

(i) If $s+t+h \leq 0$ then

$$
\max \left\{s_{j}^{2},\left(s_{n-1}+t+h\right)^{2}\right\}=s_{j}^{2} .
$$

Then

$$
g_{+}^{\prime}\left(x_{1}, x_{2}, \ldots, x_{n-1}, t\right)=\lim _{h \rightarrow 0^{+}} \frac{s_{j}^{2}-s_{j}^{2}}{h}=0
$$

(ii) If $s+t+h>0$ then

$$
\max \left\{s_{j}^{2},\left(s_{n-1}+t+h\right)^{2}\right\}=\left(s_{n-1}+t+h\right)^{2} .
$$




\section{ACCEPTED MANUSCRIPT}

Then

$$
\begin{aligned}
g_{+}^{\prime}\left(x_{1}, x_{2}, \ldots, x_{n-1}, t\right) & =\lim _{h \rightarrow 0^{+}} \frac{\left(s_{n-1}+t+h\right)^{2}-\left(s_{n-1}+t\right)^{2}}{h} \\
& =\lim _{h \rightarrow 0^{+}} \frac{h\left(2 s_{n-1}+2 t+h\right)}{h} \\
& =2 s_{j}+2 s+2 t \\
& =s+t .
\end{aligned}
$$

Thus, $g_{+}^{\prime}\left(x_{1}, x_{2}, \ldots, x_{n-1}, t\right)$ is a componentwise nondecreasing function. 